\title{
A customized home-based computerized cognitive rehabilitation platform for patients with chronic-stage stroke: study protocol for a randomized controlled trial
}

Macarena Gil-Pagés ${ }^{1,2,3,4^{*}}$ (D) Javier Solana ${ }^{1,2,3}$, Rocío Sánchez-Carrión ${ }^{1,2,3}$, Jose M. Tormos ${ }^{1,2,3}$, Antonia Enseñat-Cantallops ${ }^{1,2,3}$ and Alberto García-Molina $a^{1,2,3}$

\begin{abstract}
Background: Stroke patients usually suffer primary cognitive impairment related to attention, memory, and executive functions. This impairment causes a negative impact on the quality of life of patients and their families, and may be long term. Cognitive rehabilitation has been shown to be an effective way to treat cognitive impairment and should be continued after hospital discharge. Computerized cognitive rehabilitation can be performed at home using exercise programs that advance with predetermined course content, interval, and pace. We hypothesize that computerized rehabilitation might be improved if a program could customize course content and pace in response to patient-specific progress. The present pilot study is a randomized controlled double-blind crossover clinical trial aiming to study if chronic stroke patients with cognitive impairment could benefit from cognitive training through a customized tele-rehabilitation platform ("Guttmann, NeuroPersonalTrainer", GNPT").

Methods/design: Individuals with chronic-stage stroke will be recruited. Participants will be randomized to receive experimental intervention (customized tele-rehabilitation platform, GNPT) or sham intervention (ictus.online), both with the same frequency and duration (five sessions per week over 6 weeks). After a washout period of 3 months, crossover will occur and participants from the GNPT ${ }^{\circ}$ condition will receive sham intervention, while participants originally from the sham intervention will receive GNPT'. Patients will be assessed before and after receiving each treatment regimen with an exhaustive neuropsychological battery. Primary outcomes will include rating measures that assess attention difficulties, memory failures, and executive dysfunction for daily activities, as well as performance-based measures of attention, memory, and executive functions.
\end{abstract}

Discussion: Customized cognitive training could lead to better cognitive function in patients with chronic-stage stroke and improve their quality of life.

Trial registration: NCT03326349. Registered 31 October 2017.

Keywords: Stroke, Chronic, Randomized controlled trial, Cognitive impairment, Computerized cognitive rehabilitation

\footnotetext{
* Correspondence: macarenagil@guttmann.com

${ }^{1}$ Institut Guttmann, Institut Universitari de Neurorehabilitació, adscrit a la

Universitat Autònoma de Barcelona, Badalona, Barcelona, Spain

${ }^{2}$ Universitat Autònoma de Barcelona, Bellaterra, Cerdanyola del Vallès, Spain

Full list of author information is available at the end of the article
} 


\section{Background}

Stroke, the most common cerebrovascular disease, is a focal neurological disorder of abrupt development due to a pathological process in blood vessels [1]. There are three main types of stroke, namely transient ischemic attack, characterized by a loss of blood flow in the brain and which reverts in less than $24 \mathrm{~h}$ without associated acute infarction [2]; ischemic stroke, characterized by a lack of blood reaching part of the brain due to the obstruction of blood vessels and causing tissue damage (infarction), wherein cells die in the immediate area and those surrounding the infarction area are at risk; and a hemorrhagic stroke, where either a brain aneurysm bursts or a weakened blood vessel leaks, resulting in blood spillage into or around the brain, creating swelling and pressure, and damaging cells and tissue in the brain [3].

In 2013, according to the World Health Organization (WHO) and the Global Burden of Disease study, worldwide, there were 11-15 million people affected by stroke and almost 1.5 million deaths from this cerebrovascular disease [4, 5]. Moreover, in 2013, the total DisabilityAdjusted Life Years (years of healthy life lost while living with a poor health condition) from all strokes was $51,429,440$. In Spain, in 2011, the National Institute of Statistics reported 116,017 cases of stroke, corresponding to an incidence of 252 episodes per 100,000 inhabitants [6]. Although stroke incidence increases with advancing age, adults aged $20-64$ years comprise $31 \%$ of the total global incidence.

Stroke often results in cognitive dysfunction, and medical treatment may cause great expense on a personal, family, economic, and social level. Depending on the area of the brain affected and the severity of lesions, stroke patients may suffer cognitive impairment, and alteration in emotional and behavioral regulation [7]. Generally, cognitive impairment derived from stroke includes alterations in attention, memory, and executive function [8].

Recent reports have begun to show positive results from the use of computerized cognitive rehabilitation systems (CCRS) for stroke patients to improve attention, memory, and executive functions. Nevertheless, more research is needed to better control variables and improve training designs in order to reduce heterogeneity and increase control of the intensity and level of performance during treatments [9-12].

CCRS allow adjustment of the type of exercises administered to the specific cognitive impairment profile of each patient, but within a fixed set of possible exercises such that heterogeneity of therapy choice is minimized. This can improve studies by allowing better categorization of patient groups that execute similar training sessions in a similar range of responses [13]. Further, CCRS offers the possibility of applying cognitive rehabilitation at home, while patient adherence and performance can be monitored online, so that patients do not need to live near, lodge near, or travel to a rehabilitation center to receive therapy. Because CCRS therapy is entirely digitized, it generates objective data that can be analyzed to determine the relative effectiveness of these interventions. We hypothesize that by allowing a trained professional to oversee an automated customization program that stratifies the level of difficulty, duration, and stimulus speed of presentation, we will reduce the heterogeneity of traditional cognitive training and improve the efficacy of intervention in chronic stroke patients.

The first objective of this pilot study is to assess if chronic stroke patients with cognitive impairment could benefit from cognitive training through a customized tele-rehabilitation platform ("Guttmann, NeuroPersonalTrainer", GNPT ) [14] intended to increase the control of experimental variables (cognitive impairment profile, adherence, and performance) traditionally identified as a source of experimental heterogeneity. The study aims to assess if this benefit could translate into an improvement of the trained cognitive domains (attention, memory, and executive functions).

The second objective is focused on generalization, namely the ability to use what has been learned in rehabilitation contexts and apply it in different environments [15]. Transfer of learning is included within the concept of generalization when specifically referring to the ability to apply specific strategies to related tasks [16]. Two types of transfer have been proposed - near transfer and far transfer [17]. By near transfer we mean that, through the training of a task within a given cognitive domain, improved function in other similar, untrained tasks may be observed in the same cognitive domain. For instance, a patient who performs selective attention exercises and improves execution through the training might improve their performance in other selective attention exercises too. By far transfer we mean that training in a given cognitive domain may improve performance of tasks in other cognitive domains. Such improvement will be observable in tasks that are structurally dissimilar from the ones used in the training. For instance, if a patient performs selective attention exercises, they may also improve their performance in memory tasks.

It has been demonstrated that computerized cognitive training can lead to the phenomenon of transfer, as previously studied in stroke patients [18]. Thus, our research aims to note whether the application of patient-customized tele-rehabilitation can give rise to an improvement in other functions that are based on cognitive domains related to those that have been trained (near transfer) as well as in different ones (far transfer).

Finally, the third objective is to assess the variables of demography (age, sex, years of education) and etiology (ischemic stroke or hemorrhage) and their impact on rehabilitation outcome, given the need to understand the 
patient characteristics that may influence treatment effectiveness [19].

\section{Methods \\ Design}

The present pilot study is a double-blind, randomized, crossover clinical trial with two arms (Figs. 1 and 2, SPIRIT). Participants will be randomly assigned to either experimental intervention (GNPT) or sham intervention (ictus.online). In the first phase, participants in group A will start with the experimental intervention over a 6week period (30 sessions), and participants in group B will start with the sham intervention over the same 6week period (30 sessions). Participants will connect from their homes with their computer to the assigned intervention (experimental intervention or sham intervention). It will be indicated to them to connect once a day, from Monday to Friday, for 6 weeks. After a 3-month washout period, the second phase will commence, in which the groups will be crossed over, namely group A will receive the sham intervention and group B will receive the experimental intervention for 6 weeks each.

Evaluations will be conducted pre- and post-training by the study examiner. The examiner will be blind to the allocation of participants. Pre-testing will occur immediately before starting training (A1), and post-testing will occur immediately following both experimental and sham interventions (A2/A4) and after the 3-month washout period (A3). A follow-up neuropsychological assessment will be performed 6 months after treatment has terminated (A5).

A study researcher blinded to assessment procedure will perform the randomization of participants using the rand() function of Microsoft Excel software (Microsoft Excel 2010 for Windows), which is considered a good tool of randomization, having passed the Diehard test [20]. Each participant will have a "list entry" in our database, meaning that they will be stratified based on the two variables of interest in our study - sex (male or female) and type of stroke (ischemic or hemorrhagic). The rand() function will then assign random numbers from 0 to 1 to the stratified sample. The random numbers are ordered from lowest to highest and the first half of the list is assigned to group A and the second half to group B. A study researcher will create a letter with the information of the intervention to be performed $\left(\mathrm{GNPT}^{\circ}\right.$ or ictus.online) for each participant and will place it in a sealed envelope. The examiner will deliver the sealed envelope to each participant when the first and the third neuropsychological examinations (A1 and A3) take place.

\section{Sample size}

This is study will be an explorative pilot study. The total sample size required for a two-arm trial following some currently proposed approximation guidelines is between 24 and 70 [21]. Some recently published studies similar to this one have used sample sizes within this range

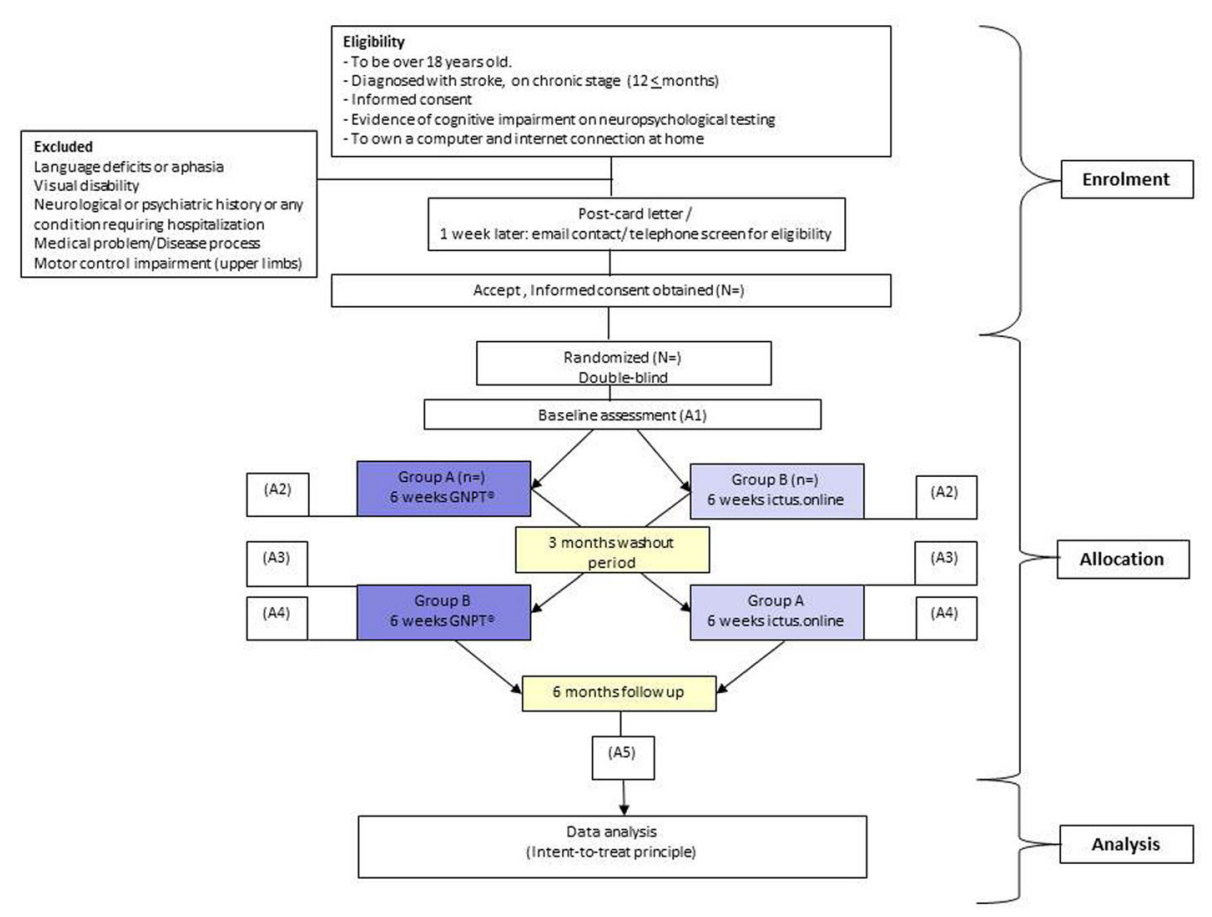

Fig. 1 Study flow diagram 


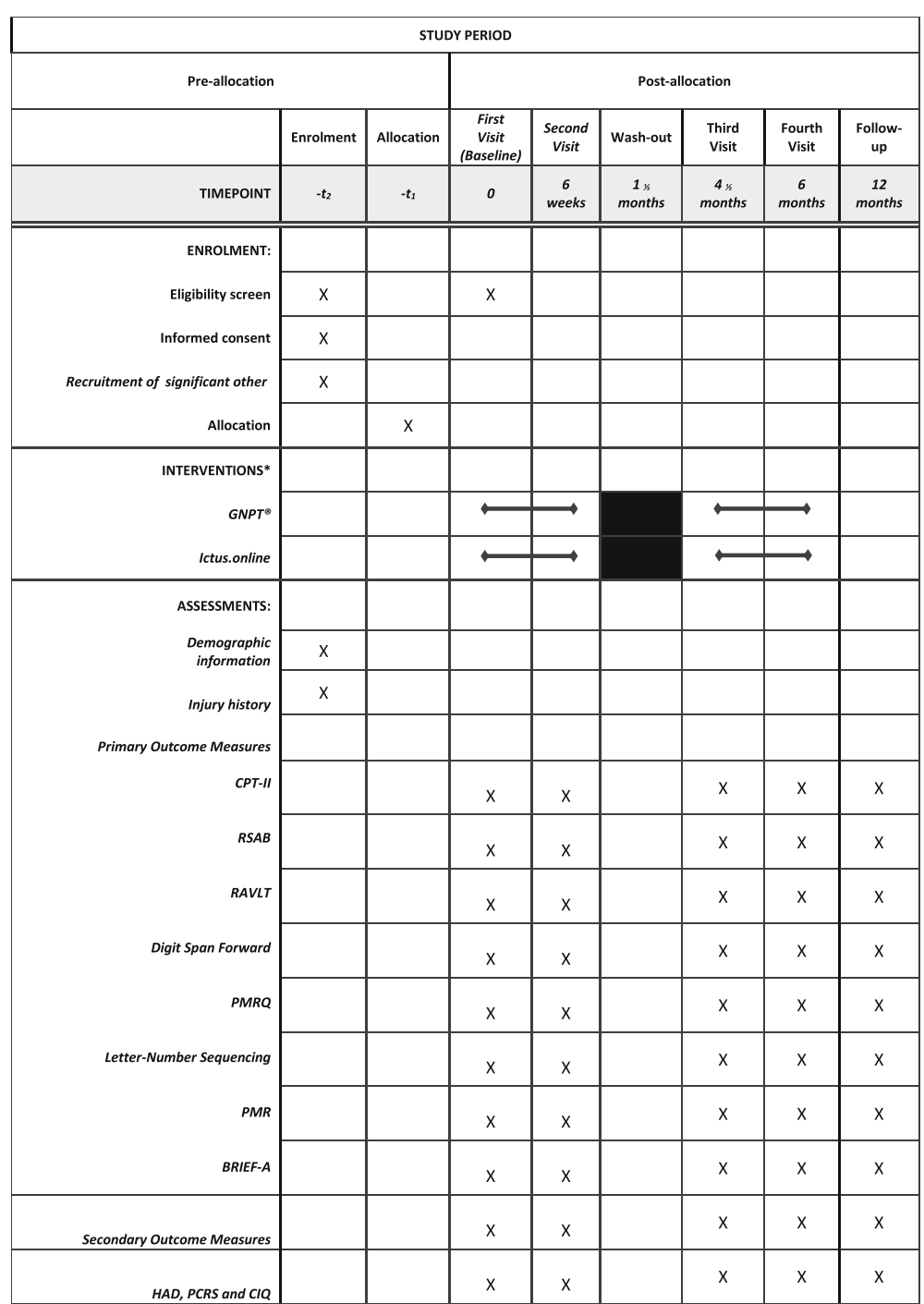

Fig. 2 SPIRIT figure: schedule of enrolment, interventions and assessments

[22-24]. The aim is to recruit 40 participants $(n=20$ per group). The results of this pilot study will be used to compute sample size and conduct a power calculation to plan a full-scale study.

\section{Participants}

Forty chronic-stage stroke patients with cognitive impairment involving alteration in attention, memory, and/ or executive functions will be recruited. The sample will be composed of former patients of Institut Guttmann who have previously received cognitive rehabilitation in the sub-acute phase of evolution.

Recruitment will include postal, email, and telephone contact with participants. Participants will be informed about the study features, goals, and implications of their participation, the duration of the study and the type of procedures that will be applied. Informed consent will be obtained in order to include them in our database. After providing informed consent to participate, participants will schedule an appointment at our center for a baseline testing session.

Inclusion criteria for the trial are (1) age over 18 years old, (2) a stroke diagnosis (ischemic stroke or hemorrhagic stroke); (3) a time of evolution of 12 months or more since stroke occurred (chronic-stage); (4) cognitive impairment confirmed by pre-intervention neuropsychological assessment; and (5) willingness to give written informed consent to participate in the study. Exclusion criteria are (1) diagnosis of language deficits or aphasia; (2) motor impairment concerning upper limbs that may incapacitate them for computer use; (3) severe alteration of the visual field or perceptual problems; (4) health status that may require 
further intervention or admission to a medical center during the study; (5) neurological or psychiatric history; or (6) substance abuse.

A neuropsychological screen evaluation to check exclusion criteria (1) and (3) will be applied. It will consist of an orientation and language test (orientation, word-repetition, visual-naming, and order comprehension subtests of Test Barcelona-R) [25]. The cutoff needed to participate in the study is $100 \%$ proficiency on these tests.

In addition, we will recruit a next-of-kin for each participant, to whom we will administer questionnaires about the impact of attention, memory, and executive function impairment on the patient's daily life at pre-training, posttraining, and follow-up testing stages.

All recruited patients to date are able to consent and understand the implications of the study. Additionally, relatives are involved in both the process of patient consent and patient performance in the study. At the time of recruitment, next-of-kin have been included and informed of the study and its characteristics and implications. This reference next-of-kin collaborates in the study by completing questionnaires about how the participant functions in daily life.

\section{Ethical considerations and informed consent}

Ethics approval has been received from the Care Ethics Committee of Fundació Institut Guttmann and the study will be conducted in accordance with the Declaration of Helsinki [26]. A neuropsychologist will explain the study to each participant by phone and in person. An information letter will be given to participants. Participants will be free to ask any question about study treatments and under no circumstances will study professionals imply that certain cognitive results will occur as a result of study participation. Further, a participant may resign from the study at any time.

The protocol follows the recommendations of the Standard Protocol Items: Recommendations for Interventional Trials Statement (SPIRIT) 2013 [27] (Additional files 1 and 2).

\section{Setting}

The study requires that five neuropsychological assessments be held in a hospital setting at the Institut Guttmann. The Institut Guttmann is a hospital and academic institution located in Badalona (Barcelona, Spain). The hospital is specialized in the medical and surgical treatment and comprehensive rehabilitation of people with spinal cord injury, acquired brain injury, or other neurological disabilities. The institute's main objective is to provide specialized, comprehensive, continuous, and personalized care, incorporating the highest levels of science and technology. In the case of patients with acquired brain injury, part of the rehabilitation includes cognitive rehabilitation, which is performed by neuropsychologists.
Participants will perform home-based interventions (GNPT ${ }^{\circ}$ and ictus.online).

\section{Intervention \\ Experimental intervention}

$\mathrm{GNPT}^{\circ}$ is a tele-rehabilitation platform that allows therapists to configure and schedule rehabilitation sessions, consisting of a set of personalized computerized cognitive exercises. The examiner from the study will perform the initial neuropsychological assessment and the results of this assessment will be stored in the GNPT ${ }^{\circ}$ system by a study researcher. The program will then calculate a cognitive profile using these results, taking into account the patient's age and educational level. Using this profile, the program will assign a set of computerized tasks to a certain day, configuring the input parameters of each task in order to personalize treatments. The neuropsychological assessment done at A1 (for group A) and A3 (for group B) will be used to establish the cognitive profile. Starting from each patient's initial cognitive capacity, therapeutic plans will be adjusted to the level of execution and adapted to the patient's evolution according to the obtained results. The adjustment is performed by GNPT $^{\circ}$ using an automated process. The difficulty level of the tasks will vary and will be adapted according to the participant's performance. Once a rehabilitation session is defined, the participant executes the assigned tasks, sending the results back to the server located at Institut Guttmann, such that therapists can asynchronously see the performance. A researcher from the study group will be exclusively dedicated to supervising this process.

The treatment consists of a set of 1-h sessions, five sessions per week during 6 weeks. A series of cognitive exercises of attention, memory, and executive functions will be conducted in each session (Fig. 3).

\section{Sham intervention}

For the sham intervention, a web platform has been developed and is available online through a the domain ictus.online (Fig. 4). This web platform has a graphic interface design similar to GNPT . After login, it allows participants to access a daily session, which presents four videos of 10 minutes each. After each video, the participant must complete a three-question quiz about the contents of the video (for example, "What animal appears in the video?"). This makes each session last approximately 1 hour in total. The difficulty level of the questions is minimal and independent of the results of the neuropsychological assessment and will remain stable regardless of the execution of the participants. The quality of execution of this condition will be monitored. 


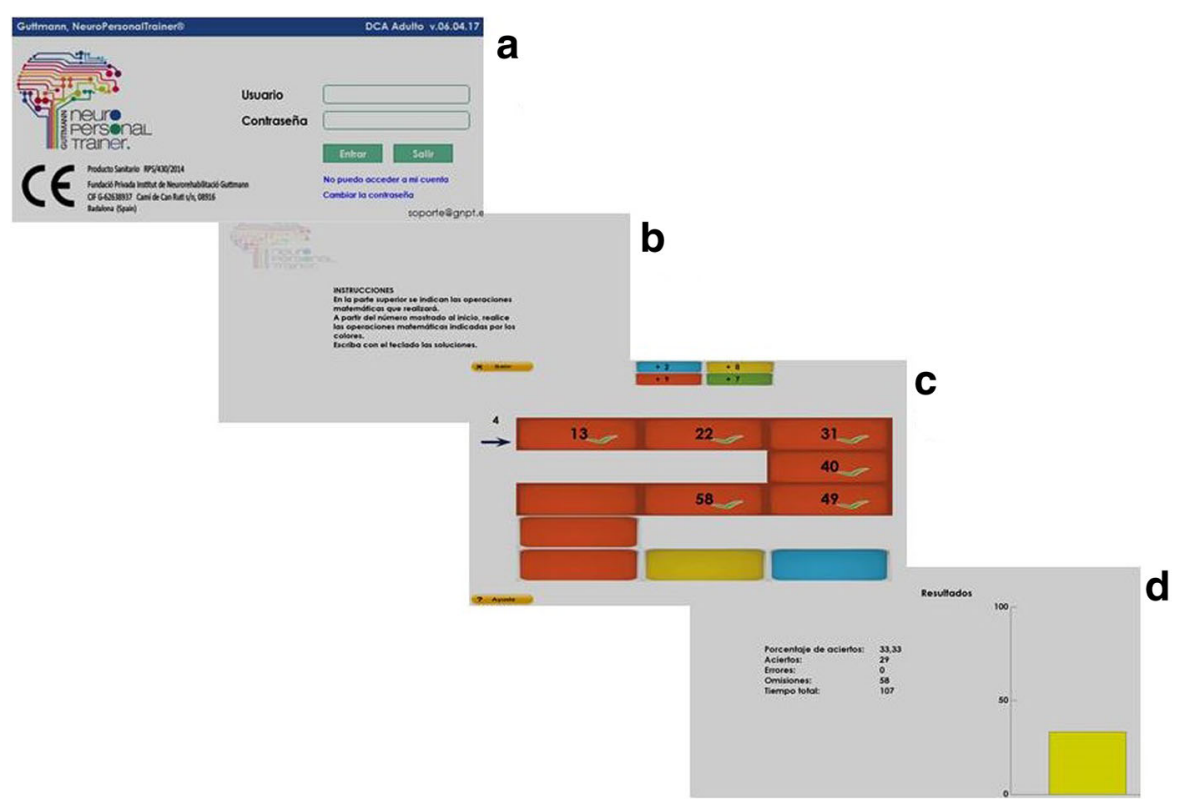

Fig. 3 Experimental intervention (GNPT ) screenshots. a To access to the platform, the user must enter their username and password. $\mathbf{b}$ Each exercise begins with an instruction screen. $\mathbf{c}$ Example of a cognitive rehabilitation exercise. $\mathbf{d}$ At the end of each exercise, a results screen is displayed

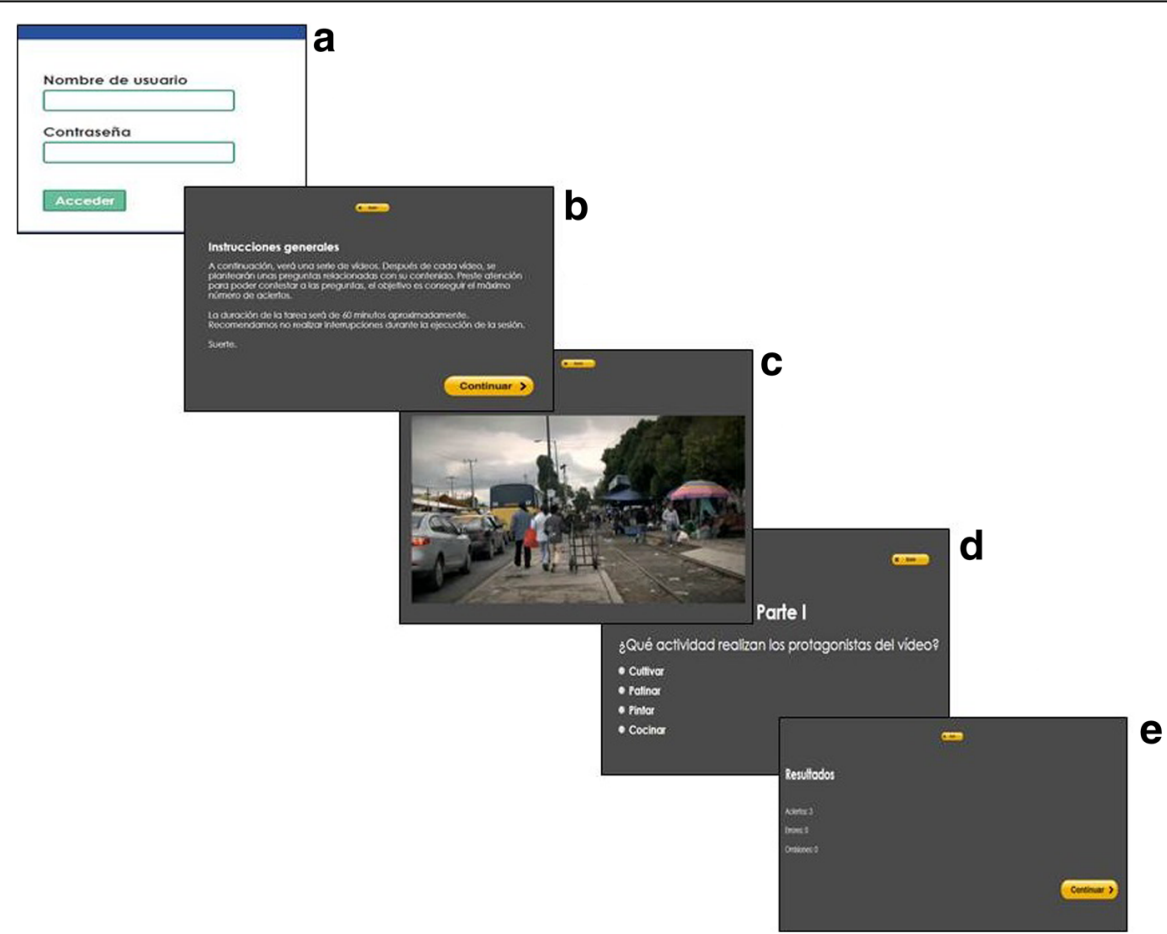

Fig. 4 Sham intervention (ictus.online) screenshots. a To access to the platform, the user must enter their username and password. b Each exercise begins with an instruction screen. $\mathbf{c}$ The user watches a 10-min video. $\mathbf{d}$ When finished, the user accesses a three-question quiz with four response options. e When the quiz is finished, a results screen is displayed. In each session, three videos with their corresponding quiz are presented 
Both the experimental intervention group and the sham intervention group will receive a username and password for logging in.

As adherence strategies, the organizers will offer flexible schedules to perform the exercises, phone calls to confirm appointment dates, and periodic progress reports to keep participants informed on their personal progress in the study (i.e., how much work they have completed, how much longer the study will last, etc.). Additionally, connection frequency will be supervised. To reduce risk, a member of the organizing team will contact the participants if they do not perform any exercise at any of the platforms of treatment for 3 consecutive days $\left(\mathrm{GNPT}^{\circ}\right.$ or ictus.online) and will reinforce treatment compliance. Attrition will be considered when (1) presence of a new neurological condition appears during the study, or (2) loss of contact with participants occurs during the study and follow-up.

\section{Measures}

Demographic information (age, sex, marital status, occupation, education level) and injury history (time of evolution, type of stroke) from all participants will be collected. Whether they have received or are currently receiving any kind of neuropsychological or functional rehabilitation at the moment of joining the study will be also recorded. The information collection method will be a personal interview. A comprehensive neuropsychological battery will be used to assess the participants. This battery is comprised of performance-based measures to assess attention (Conners Continuous Performance, CPT-II) [28], Trail Making Test A and B [29]), memory (Digit Span forward from the Wechsler Adult Intelligence Scale (WAIS-III) [30], Rey Auditory Verbal Learning Test [31]), speed of processing (Digit Symbol-Coding test from WAIS-III), visuoconstruction (Block Design test from WAIS-III), and executive functions (Digit Span backwards and LetterNumber Sequencing from WAIS-III, Stroop Color and Word test [32], Wisconsin Card Sorting Test - computerized version [33], and a Spanish phonemic fluency test PMR [34]). Administered rating measures (Rating Scale for Attentional Behavior (RSAB) [35]; Prospective and Retrospective Memory Questionnaire (PRMQ) [36]; Behavior Rating Inventory of Executive Function - Adult Version (BRIEF-A)) [37] will be administered alongside the performance-based measures. All these rating measures will be applied to the patient (self-report) and their recruited next-of-kin (informant-report). Finally, a psychological well-being self-rating scale (Hospital Anxiety and Depression Scale [38]), Patient Competency Rating Scale [39], and the Community Integration Questionnaire [40] will be administered. The Hospital Anxiety and Depression scale will be used to detect participants with emotional disorders. The Patient Competency Rating Scale comprises 30 items, spanning a range of everyday situations and behaviors, and for each item, the patient must make judgment about their own level of competency. The Community Integration Questionnaire is a self-rating scale developed to measure community integration that comprises three subscales (home integration, social integration, and productive activities). These rating measures have been used before in other studies involving stroke patients [41-43].

\section{Primary outcome measures}

The primary outcome measures are performance-based measures and rating measures of attention, memory, and executive functions. Since they are different cognitive domains, we need to use different measures for attention (two primary outcomes: the CPT-II and RSAB), memory (Rey Auditory Verbal Learning Test, Digit Span forward from WAIS-III, and the PRMQ), and executive functions (Spanish phonemic fluency test - PMR, Digit Span backward, and the Letter-Number Sequencing Subtests from the WAIS-III and the BRIEF-A). CPT-II is a taskoriented computerized assessment of sustained attention; the Rey Auditory Verbal Learning Test assesses short- and long-term verbal memory and recognition; the PMR test assesses capacity of word generation according to an initial letter (P, M and R); the Digit Span forward test measures the span of immediate verbal recall; Digit Span backward and Letter Number assess working memory; and the RSAB comprises 14 items aimed to identify attentional difficulties on daily routines. The PRMQ includes 16 items, and was designed to evaluate prospective and retrospective memory in the short and long term. The BRIEF-A is designed to assess executive function behaviors on day-to-day activities.

In order to reduce the number of analyses, we will create composite cognitive indices - one for attention, another for memory, and another for executive functions, using sub-scores from the various performance-based measurements of the primary outcome. Composite cognitive indices will include sub-scores on CPT-II, namely number of omissions, number of commissions, and hit of response for attention; sub-scores on Rey Auditory Verbal Learning Test (Trials 1-5 recall, long-delay free recall and recognition) and Digit Span forward from WAIS-III (immediate recall of a series of numbers) for memory; and scores on Spanish phonemic fluency test PMR (phonemic word fluency), Digit Span backward from the WAIS-III (immediate recall of a sequence of numbers in reverse order in which it was presented), and Letter-Number Sequencing Subtests from the WAIS-III (recall of progressively longer lists of intermixed letters and numbers in alphabetical and then numerical order) for executive functions. 


\section{Secondary outcome measures}

Secondary outcomes are the Digit Symbol-Coding and the Block Design Subtests from the WAIS-III, Trail Making Test A and B (TMT-A and TMT-B), Wisconsin Card Sorting Test, and Stroop Color and Word Test. The Digit Symbol-Coding assesses the speed of processing; Block Design evaluates visuoconstruction and planning; TMT-A measures visual attention, while TMT-B measures task-switching; the Wisconsin Card Sorting Test is a measure of cognitive flexibility; and the Stroop Color and Word test is used as a measure of inhibitory control.

\section{Statistical analysis}

Analysis Data analysis will be based on the intentionto-treat principle, which means that all participants who were randomized will be included in the final analysis, regardless of their adherence to any of the interventions. To avoid the problems derived from missing data (no attendance to neuropsychological assessments), blank spots will be filled with missing data imputation technique last value carried forward.

In order to detect changes in the variables recorded for the primary outcome and secondary outcome, a withinsubject comparison pre-/post-intervention in both groups after both interventions will be made, as well as comparisons between groups (group A and group B).

Our null hypothesis is that a difference between baseline scores (A1 for group A and A3 for group B) and scores post-treatment (A2 for group A and A4 for group B) will not be significantly different from zero. Therefore, the Kolmogorov-Smirnov test will be used to examine the data for normality, and either ANOVA (parametric test) or Kruskal-Wallis (non-parametric test) will be used to analyze the measurements. A significance level of $95 \%$ will be used $(P<0.05$ cutoff). Demographic (age, sex, years of education) and etiologic characteristics (ischemic stroke or hemorrhage) will be described using descriptive statistics for each group (group A and group B). The Bonferroni method will be applied to adjust the overall level of significance for multiple outcomes.

Alternatively, composite indices will be created by transforming raw neuropsychological test scores into $\mathrm{z}$ scores using published normative data. Domains will be created by averaging the $\mathrm{z}$ scores for each test within the domain $(M=0 ; S D=1)$. This system has been used before in similar studies [44, 45].

If the sample size of this pilot study allows, a longitudinal mixed model will be applied.

Data analysis will be performed by an analyst outside the study with the statistical analysis software R and appropriate packages ("lmm" and "psych") [46].

\section{Discussion}

The first objective of this research is to study the effectiveness of a patient-customized computerized cognitive training program (GNPT) as a home-based cognitive stimulation tool for stroke patients in the chronic stage of recovery. We hypothesize that training cognitive functions of attention, memory, and executive functions with GNPT $^{\circ}$ could lead to better function of those cognitive domains post-therapy. Furthermore, we want to study whether customized cognitive training could lead to an improvement in other contexts different from the examination tests, improving performance in both related (near transfer) and unrelated (far transfer) tasks. Finally, we want to study the impact of variables such as demography and etiology on rehabilitation outcome.

The strengths of our study include that it is designed to (1) be easily replicated in new patient cohorts, (2) minimize unintended variability by reducing intervention heterogeneity, (3) adjust the type and difficulty of exercises to the specific cognitive impairment profile of patients, and (4) monitor adherence and performance of each exercise during the whole treatment.

Furthermore, our study focuses on targeting cognitive rehabilitation toward the improvement of the quality of life of the patient. In this sense, we include in our primary outcome measures an assessment of functioning in daily life, before, during, and after the performance of both interventions. Additionally, our design is longitudinal, which offers the possibility of studying the maintenance of the results over time (over 3 and 6 months). The timing of the primary outcome is 3 months. The purpose of the follow-up at 6 months is to check the evolution of patients once they have stopped receiving any type of intervention.

Furthermore, the execution of both interventions will be remotely monitored and we will be able to record the quantity and quality of the execution of the participants. Nevertheless, we consider a limitation the fact that we will not be able to control the environmental conditions in which participants perform the tasks. Another limitation is that the battery assessment will be the same throughout the study, which might create a learning effect and/or ceiling effect in some evaluation tasks.

\section{Trial status}

This article was submitted on January 3, 2018. Recruitment is ongoing.

\section{Additional files}

Additional file 1: SPIRIT 2013 Checklist: Recommended items to address in a clinical trial protocol and related documents*. (DOC $121 \mathrm{~kb}$ )

Additional file 2: CONSORT 2010 checklist of information to include when reporting a randomised trial*. (DOC $217 \mathrm{~kb}$ ) 


\section{Abbreviations}

CPT-II: Conners Continuous Performance Test; A1: first assessment; A2: second assessment; A3: third assessment; A4: fourth assessment; A5: fifth assessment; BRIEF-A: Behavior Rating Inventory of Executive Function-Adult; GNPT": "Guttmann, NeuroPersonalTrainer"; PMR: Spanish phonemic fluency test; PRMQ: Prospective and Retrospective Memory Questionnaire; RSAB: Rating Scale for Attentional Behavior; TMT: Trail Making Test; WAIS: Wechsler Adult Intelligence Scale

\section{Acknowledgements}

The authors are grateful to Dr. Adam G. Schrum for providing writing assistance and general support.

\section{Funding}

This study is funded by BIOCAT La Fundació BioRegió de Catalunya - Registry Number: COM15-1-0004 and Fundació Bancària "la Caixa" - Obra Social "la Caixa" Project number: LCF/PR/PR16/11110004.

\section{Availability of data and materials}

\section{Not applicable.}

\section{Authors' contributions}

AGM: trial conception, study design and reviewing of manuscript. MGP: study design, drafting and reviewing of manuscript. JS: advice in telecommunications engineering and reviewing of manuscript. RSC: refinement of the study and reviewing of manuscript. JMT: study design and reviewing of manuscript. AEC: coordination of the study and reviewing of manuscript. All authors read and approved the final manuscript.

\section{Ethics approval and consent to participate}

Ethics approval has been received from Care Ethics Committee of Fundació Institut Guttmann. Informed consent from all participants in the study will be obtained.

\section{Consent for publication}

Not applicable.

\section{Competing interests}

The GNPT is partly property of Institut Guttmann. JS, AGM, RSC, and JMT have been involved in the development of the GNPT'.

\section{Publisher's Note}

Springer Nature remains neutral with regard to jurisdictional claims in published maps and institutional affiliations.

\section{Author details}

${ }^{1}$ Institut Guttmann, Institut Universitari de Neurorehabilitació, adscrit a la Universitat Autònoma de Barcelona, Badalona, Barcelona, Spain. ${ }^{2}$ Universitat Autònoma de Barcelona, Bellaterra, Cerdanyola del Vallès, Spain. ${ }^{3}$ Fundació Institut d'Investigació en Ciències de la Salut Germans Trias i Pujol, Badalona, Barcelona, Spain. ${ }^{4}$ Departament de Psicologia Clínica i de la Salut, Universitat Autònoma de Barcelona, Bellaterra, Cerdanyola del Vallès, Spain.

\section{Received: 3 January 2018 Accepted: 6 March 2018}

\section{Published online: 22 March 2018}

\section{References}

1. Donaghy M. Brain's Diseases of the Nervous System. 12th ed. New York: Oxford University Press; 2009

2. Lezak MD, Howieson DB, Bigler ED, Tranel D. Neuropsychological Assessment. 5th ed. New York: Oxford University Press; 2012.

3. Sacco R, Kasner S, Broderick J, Caplan L, Connors J, Culebras A, Elkind M, George M, Hamdan A, Higashida R, Hoh B, Janis L, Kase C, Kleindorfer D, Lee J, Moseley M, Peterson E, Turan T, Valderrama A, Vinters H. An updated definition of stroke for the 21st century: a statement for healthcare professionals from the American Heart Association/American Stroke Association. Stroke. 2013;44:2064-89.

4. Krishnamurthi R, Moran AE, Feigin VL, Barker-Collo S, Norrving B, Mensah GA, Taylor S, Naghavi M, Forouzanfar MH, Nguyen G, Johnson CO, Vos T, Murray CJ, Roth GA. Stroke prevalence, mortality and disability-adjusted life years in adults aged 20-64 years in 1990-2013: Data from the Global Burden of Disease 2013 Study. Neuroepidemiology. 2015;45(3):190-202. https://doi. org/10.1159/000441098

5. World Health Organization. Regional Office for the Eastern Mediterranean. 2017. http://www.emro.who.int/health-topics/stroke-cerebrovascularaccident/index.html. Accessed 3 Oct 2017.

6. Brea A, Laclaustra M, Martorell E, Pedragosa À. Epidemiology of cerebrovascular disease in Spain. Clínica e Investigación en Arteriosclerosis. 2013;25(Suppl 5):211-7.

7. Teasell R, Hussein N, Viana R, Madady M, Donaldson S, McClure A, Richardson M. Clinical Consequences of Stroke. Stroke Rehabilitation Clinician Handbook. 2016. http://www.ebrsr.com/clinician-handbook. Accessed 1 May 2017.

8. Ma VY, Chan L, Carruthers KJ. Incidence, prevalence, costs and impact on disability of common conditions requiring rehabilitation in the United States: stroke, spinal cord injury, traumatic brain injury, multiple sclerosis, osteoarthritis, rheumatoid arthritis, limb loss and back pain. Arch Phys Med Rehabil. 2014;95:986-95.

9. Prokopenko S, Mozheyko E, Petrova M, Koryagina T, Kaskaeva D, Chernykh T, Shvetzova I, Bezdenezhnih A. Correction of post-stroke cognitive impairments using computer programs. J Neurol Sci. 2013:325:148-53.

10. van de Ven $R$, Schmand $B$, Groet $E$, Veltman D, Murre J. The effect of computer-based cognitive flexibility training on recovery of executive function after stroke: rationale, design and methods of the TAPASS study. BMC Neurol. 2015;15:144. https://doi.org/10.1186/s12883-015-0397-y

11. Bogdanova Y, Yee M, Ho V, Cicerone K. Computerized cognitive rehabilitation of attention and executive function in acquired brain injury. J Head Trauma Rehabil. 2016:31:419-33.

12. Poulin V, Korner-Bitensky N, Dawson D, Bherer L. Efficacy of executive function interventions after stroke: a systematic review. Top Stroke Rehabil. 2012;19:158-71.

13. Solana J, Cáceres C, García-Molina A, Chausa P, Opisso E, Roig-Rovira T, Ernestina M, Tormos-Muñoz JM, Gómez EJ. Intelligent Therapy Assistant (ITA) for cognitive rehabilitation in patients with acquired brain injury. BMC Med Inform Decis Mak. 2014;14:58.

14. Solana J, Cáceres C, García-Molina A, Opisso E, Roig T, Tormos JM, Gómez EJ. Improving brain injury cognitive rehabilitation by personalized telerehabilitation services: Guttmann Neuropersonal Trainer. IEEE J Biomed Health Inf. 2015;19(1):124-31.

15. Zelinski E. Far transfer in cognitive training of older adults. Restor Neurol Neurosci. 2009;27(Suppl 5):455-71.

16. Toglia JP. Generalization of treatment: a multicontext approach to cognitive perceptual impairment in adults with brain injury. Am J Occup Ther. 1991; 45(6):506-16.

17. Barnett SM, Cecci SJ. When and where do we apply what we learn? A taxonomy for transfer. Psychol Bull. 2002;128(4):612-37.

18. Van de Ven RM, Murre MJ, Veltman DJ, Schmand BA. Computer-based cognitive training for executive functions after stroke: a systematic review. Front Hum Neurosci. 2016:10:150

19. Cicerone K, Langenbahn D, Braden C, Malec J, Kalmar K, Fraas M, Felicetti T, Laatsch L, Harley J, Bergquist T, Azulay J, Cantor J, Ashman T. Evidencebased cognitive rehabilitation: updated review of the literature from 2003 through 2008. Arch Phys Med Rehabil. 2011;92:519-30.

20. Microsoft Support: Description of the RAND Function in Excel. 2017. https:// support.microsoft.com/en-hk/help/828795/description-of-the-rand-functionin-excel. Accessed 5 Feb 2018.

21. Whitehead A, Julious S, Cooper C, Campbell M. Estimating the sample size for a pilot randomised trial to minimise the overall trial sample size for the external pilot and main trial for a continuous outcome variable. Stat Methods Med Res. 2015;25:1057-73.

22. De Luca R, Calabrò R, Gervasi G, De Salvo S, Bonanno L, Corallo F, De Cola $M$, Bramanti P. Is computer-assisted training effective in improving rehabilitative outcomes after brain injury? A case-control hospital-based study. Disabil Health J. 2014;7:356-60.

23. Westerberg H, Jacobaeus $H$, Hirvikoski T, Clevberger $P$, Östensson M, Bartfai A, Klingberg T. Computerized working memory training after stroke - A pilot study. Brain Inj. 2007;21:21-9.

24. Björkdahl A, Åkerlund E, Svensson S, Esbjörnsson E. A randomized study of computerized working memory training and effects on functioning in everyday life for patients with brain injury. Brain Inj. 2013:27:1658-65.

25. Peña-Casanova J. Normalidad, Semiología y Patología Neuropsicológicas. Programa Integrado de Exploración Neuropsicológica. Test Barcelona Revisado. 2nd ed. Barcelona: Masson; 2005. 
26. World Medical Association Declaration of Helsinki. Ethical principles for medical research involving human subjects. JAMA. 2013;310(20):2191-4.

27. Chan A-W, Tetzlaff JM, Altman DG, Laupacis A, Gøtzsche PC, Krleža-Jerić K, Hróbjartsson A, Mann H, Dickersin K, Berlin J, Doré C, Parulekar W, Summerskill W, Groves T, Schulz K, Sox H, Rockhold FW, Rennie D, Moher D. SPIRIT 2013 statement: defining standard protocol items for clinical trials. Ann Intern Med. 2013;158:200-7.

28. Conners CK. Conners' Continuous Performance Test II: Computer Program for Windows Technical Guide and Software Manual. North Tonwanda: MutliHealth Systems; 2000.

29. Tombaugh T. Trail Making Test A and B: Normative data stratified by age and education. Arch Clin Neuropsychol. 2004;19(2):203-14. https://doi.org/ 10.1016/s0887-6177(03)00039

30. Wechsler D. WAIS-III. Administration and Scoring Manual. San Antonio: The Psychological Association; 1997.

31. Schmid M. Rey Auditory and Verbal Learning Test: A Handbook. Los Angeles: Western Psychological Services; 1996.

32. Golden CJ. Test of Colors and Words. Madrid: Tea Editions; 2001

33. Roig-Fusté JM. Prueba de clasificación de cartas. Barcelona: MTR; 2001.

34. Artiola i Fortuny L, Hermosillo Romo D, Heaton RK, Pardee RE III. Manual de Normas y Procedimientos para la Batería Neuropsicológica en Español. Tucson: mPress; 1999.

35. Ponsford J, Kinsella G. The use of a rating scale of attentional behavior. Neuropsychol Rehabil. 1991;1(4):241-57.

36. Crawford JR, Smith G, Maylor EA, Della Sala S, Logie RH. The Prospective and Retrospective Memory Questionnaire (PRMQ): normative data and latent structure in a large non-clinical sample. Memory. 2003;11(3):261-75.

37. Roth RM, Isquith PK, Goia GA. BRIEF-A: Behavior Rating Inventory of Executive Function - Adult version. Lutz: Psychological Assessment Resources, Inc.; 2005.

38. Zigmond AS, Snaith RP. The Hospital Anxiety and Depression Scale. Acta Psychiatr Scand. 1983;67:361-70.

39. Prigatano GP, Fordyce DJ, Zeiner HK, Roueche JR, Pepping M, Wood BC Neuropsychological Rehabilitation after Brain Injury. Baltimore: Johns Hopkins University Press; 1986.

40. Willer B, Rosenthal M, Kreutzer JS, Gordon WA, Rempel R. Assessment of community integration following rehabilitation for traumatic brain injury. J Head Trauma Rehabil. 1993;8(2):75-87.

41. Fischer S, Trexler LE, Gauggel S. Awareness of activity limitations and prediction of performance in patients with brain injuries and orthopedic disorders. J Int Neuropsychol Soc. 2004;10(2):190-9.

42. Gauggel S, Peleska B, Bode RK. Relationship between cognitive impairments and rated activity restrictions in stroke patients. J Head Trauma Rehabil. 2000;15(1):710-23.

43. Lee H, Lee $Y$, Choi H, Pyun SB. Community integration and quality of life in aphasia after stroke. Yonsei Med J. 2015;56:1694-702.

44. Habeck C, Razlighi Q, Gazes Y, Barulli D, Steffener J, Stern Y. Cognitive reserve and brain maintenance: orthogonal concepts in theory and practice. Cereb Cortex. 2017;27:3962-9.

45. Steward K, Kennedy R, Novack T, Crowe M, Marson D, Triebel K. The role of cognitive reserve in recovery from traumatic brain injury. J Head Trauma Rehabil. 2018;33(1):E18-27.

46. R Core Team. R: A Language and Environment for Statistical. Vienna: Computing. R Foundation for Statistical Computing; 2017. https:/www.R-project.org. Accessed 27 Sept 2017

\section{Submit your next manuscript to BioMed Central and we will help you at every step:}

- We accept pre-submission inquiries

- Our selector tool helps you to find the most relevant journal

- We provide round the clock customer support

- Convenient online submission

- Thorough peer review

- Inclusion in PubMed and all major indexing services

- Maximum visibility for your research

Submit your manuscript at www.biomedcentral.com/submit 